\title{
TEARING MODES GROWTH RATE AMPLIFICATION DUE TO FINITE CURRENT RELAXATION
}

\author{
F. E. M. SilveirA
}

Centro de Ciências Naturais e Humanas, Universidade Federal do ABC, Rua Santa Adélia, 166, Bairro Bangu, CEP 09210-170, Santo André, SP, Brazil

correspondence: francisco.silveira@ufabc.edu.br

ABSTRACT. In this work, we explore the influence of perturbative wavelengths, shorter than those usually considered, on the growth rate $\gamma$ of the tearing modes. Thus, we adopt an extended form of Ohm's law, which includes a finite relaxation time for the current density, due to inertial effects of charged species. In the long wavelength limit, we observe the standard $\gamma$ of the tearing modes. However, in the short wavelength limit, we show that $\gamma$ does not depend on the fluid resistivity any longer. Actually, we find out that $\gamma$ now scales with the electron number density $n_{\mathrm{e}}$ as $\gamma \sim n_{\mathrm{e}}^{-3 / 2}$. Therefore, through a suitable combination of both limiting results, we show that the standard $\gamma$ can be substantially amplificated, even by moderate shortenings of perturbative wavelengths. Further developments of our theory may contribute to the explanation of the fast magnetic reconnection of field lines, as observed in astrophysical plasmas.

KEYWORDS: tearing modes; current relaxation; magnetic islands; magnetic reconnection.

\section{INTRODUCTION}

The most violent instabilities in magnetically confined plasmas are the so-called ideal hydromagnetic instabilities, which are driven by current and pressure gradients 1.3. As a matter of fact, the condition of a vanishingly small resistivity imposes a constraint on the allowed perturbed motions in the fluid. Actually, the electric field in a frame moving with an ideal plasma has to vanish. Thus, the magnetic flux through any surface moving with that fluid has to remain constant. Therefore, the perturbed magnetic field lines cannot slip with respect to the perturbed flow lines in an ideal plasma. According to Faraday's law, the magnetic field remains frozen inside the ideal fluid or obeys the frozen-in law.

The frozen-in law and ideal instabilities are wellknown subjects in plasma physics. They are discussed in many textbooks, from the introductory to the advanced levels $4-8$. The ideal instabilities are not only violent in the sense that they can destroy a given equilibrium configuration, which, in this case, is determined by the balance of the pressure gradient with the Lorentz force. Their growth rate in time can typically achieve very large values as well. This feature has lead to a great effort to achieve control of the relevant physical parameters, which characterize actual confinement configurations, such that those structures can remain stable to ideal modes 913 .

It is true that a finite electric resistivity provokes a decrease on the current gradient, which drives the ideal modes. However, it should be noted that dissipative effects can relax constraints in the ideal fluid as well. Thus, states with lower potential energy can become accessible to the system and new instabilities can emerge. In particular, a finite plasma resistivity relaxes the frozen-in condition. Therefore, magnetic field lines can break-up into thin filaments. These structures were coined magnetic islands [14 18.

Magnetic islands play a central role in the physics of magnetic confinement configurations. They usually grow in a time scale much longer than the Alfvén time scale and attain a saturated size when the linear free energy, which was available to drive the change in the topology of the magnetic field lines, vanishes. If the saturated size of the magnetic islands is comparable with the radius of the plasma column in a tokamak, then heat flow across the field lines is essentially replaced by the much faster flow along the lines. When the saturated islands are sufficiently large to touch each other or the material limiter inside the vacuum chamber, a disruption of the plasma column can occur and the chamber can become subjected to strong mechanical stresses [19 23].

In astrophysical plasmas, magnetic islands play an equally relevant role. Magnetic energy can be converted into kinetic energy, thermal energy, and particle acceleration due to the modification of the magnetic topology in highly conducting plasmas. This phenomenon was dubbed the magnetic reconnection. Quite interestingly, the magnetic reconnection is observed to occur much faster than theoretically predicted in most astrophysical processes. For instance, solar flares eventuate several orders of magnitude faster than predicted, even by including kinetic, sthocastic, and turbulence effects into the topological dynamics [24 29]. This issue suggests the possibility that there is still enough room to explore the most basic mechanisms, which underly the formation of magnetic islands. The resistive instability that is responsible to form magnetic islands is known as the tearing instability.

The linear theory of the tearing modes was originally 
discussed by Furth, Killeen, and Rosenbluth 30, and further developed by many authors 31 35. A linear perturbation is effected on a given static state of equilibrium of an infinite ideal plasma, which contains a thin plane slab, with a small resistivity $\eta$. Therefore, on the assumption of the usual Ohm's law, which establishes that the electric field in a frame moving with the fluid is equal to the product of the plasma resistivity with the current density, one finds that the growth rate $\gamma$ of the tearing modes scales with $\eta$ as $\gamma \sim \eta^{3 / 5}$. This is the central result of the standard theory of the tearing modes.

In this work, we investigate the influence of perturbative wavelengths, shorter than those usually considered, on the growth rate of the tearing modes. Thus, we adopt an extended form of Ohm's law, which includes a finite relaxation time for the current density, due to inertial effects of charged species. In the long wavelength limit, we observe the standard growth rate of the tearing modes. However, in the short wavelength limit, we can see that the growth rate does not depend on the fluid resistivity any longer. Actually, we can find out that $\gamma$ now scales with the electron number density $n_{\mathrm{e}}$ as $\gamma \sim n_{\mathrm{e}}^{-3 / 2}$. Therefore, through a suitable combination of both limiting results, we show that the standard growth rate of the tearing modes can be substantially amplificated, even by moderate shortenings of perturbative wavelengths. Further developments of our theory may contribute to the explanation of the fast magnetic reconnection of field lines, as observed in astrophysical plasmas.

\section{BASIC EQUATIONS AND LINEAR ANALYSIS}

Let us start by considering an infinite plasma, in the presence of a magnetic field $\vec{B}$, flowing with a velocity $\vec{V}$. By adopting Cartesian coordinates $(x, y, z)$, we assume that

$$
\vec{B}=\hat{z} B_{\| 0}-\hat{z} \times \nabla \Psi, \quad \vec{V}=-\hat{z} \times \nabla \Phi,
$$

where the flux functions $\Psi$ and $\Phi$ depend only on $x$ and $y$ (of course, they are allowed to depend on the time $t$ as well), and $B_{\| 0}$ is a constant. While writing (1), it should be noted that the conditions on the absence of magnetic monopoles, $\nabla \cdot \vec{B}=0$, and incompressibility, $\nabla \cdot \vec{V}=0$, are automatically satisfied.

In this work, we aim to explore the influence of perturbative wavelengths, shorter than those usually considered, on the growth rate of tearing modes. Therefore, we adopt an extended form of Ohm's law (a still more general formula should include ion and electron pressure gradients, as well as the Hall effect [7, 36, 40, however, those terms are not relevant for our purposes),

$$
\vec{E}+\vec{V} \times \vec{B}=\eta\left(1+\tau_{\mathrm{C}} \frac{\partial}{\partial t}\right) \vec{J}
$$

where $\vec{E}, \vec{J}$, and $\eta$ are the electric field, current density, and electric resistivity, respectively. For singly ionized, approximately neutral, resistive plasmas, $\tau_{\mathrm{C}}=$ $m_{\mathrm{e}}\left(n_{\mathrm{e}} e^{2} \eta\right)^{-1}$, with $m_{\mathrm{e}}, n_{\mathrm{e}}$, and $e$ standing for the electron mass, number density and charge, respectively. As a matter of fact, $\tau_{\mathrm{C}}$ must be interpreted as the finite relaxation time for the current density, due to inertial effects of charged species. Actually, if the electromagnetic field is suddenly removed from the presence of the fluid, then $\sqrt{2}$ shows that $\vec{J}(t)=\vec{J}(0) \mathrm{e}^{-\tau_{\mathrm{C}}^{-1} t}$. This means that the initial current $\vec{J}(0)$ damps off in the fluid, in a time interval of the order of the scale $\tau_{\mathrm{C}}$. At sufficiently long wavelengths, characterizing the fields, inertial effects are negligible, $\tau_{\mathrm{C}} \rightarrow 0$, and the initial current damps off instantaneously. In this case, 22 recovers the more usual form of Ohm's law, $\vec{E}+\vec{V} \times \vec{B}=\eta \vec{J}$.

Equations 11 imply $\vec{V} \times \vec{B}=-\hat{z} \vec{V} \cdot \nabla \Psi-\hat{z} \times \vec{V} B_{\| 0}$. Now, by combining the first of (1) with the Faraday and Ampère laws (the displacement current is neglected in the Ampère-Maxwell law: the hydromagnetic approximation), we obtain

$$
\vec{E}=-\nabla \chi-\hat{z} \frac{\partial \Psi}{\partial t}, \quad \vec{J}=-\hat{z} \frac{\nabla^{2} \Psi}{\mu_{0}},
$$

where $\chi$ and $\mu_{0}$ are the electric potential and vacuum magnetic permeability (a diamagnetic plasma is assumed), respectively.

By substituting the expanded $\vec{V} \times \vec{B}$ and 3 in (2), the $z$-component of the latter provides an expression for the time evolution of the magnetic flux,

$$
\hat{z} \cdot \nabla \chi+\left(\frac{\partial}{\partial t}+\vec{V} \cdot \nabla\right) \Psi=\frac{\eta}{\mu_{0}}\left(1+\tau_{\mathrm{C}} \frac{\partial}{\partial t}\right) \nabla^{2} \Psi
$$

We seek an expression for the time evolution of the flow flux. Therefore, we consider Euler's equation (an inviscid fluid is assumed),

$$
\left(\frac{\partial}{\partial t}+\vec{V} \cdot \nabla\right) \vec{V}=\frac{-\nabla P+\vec{J} \times \vec{B}}{\rho_{0}},
$$

where $P$ and $\rho_{0}$ are the hydrodynamic pressure and (constant and uniform) mass density, respectively. By taking the curl of the relevant terms in (5) (of course, $\nabla \times \nabla P=0)$, we obtain

$$
\begin{gathered}
\nabla \times\left(\frac{\partial}{\partial t}+\vec{V} \cdot \nabla\right) \vec{V}=-\hat{z}\left(\frac{\partial}{\partial t}+\vec{V} \cdot \nabla\right) \nabla^{2} \Phi, \\
\nabla \times(\vec{J} \times \vec{B})=-\frac{\nabla\left(\nabla^{2} \Psi\right) \times \nabla \Psi}{\mu_{0}},
\end{gathered}
$$

where, again, we use (1) and Ampère's law. Finally, by combining the $z$-components of (5) and (6), we arrive at the sought expression,

$$
\left(\frac{\partial}{\partial t}+\vec{V} \cdot \nabla\right) \nabla^{2} \Phi=\frac{\hat{z} \cdot \nabla\left(\nabla^{2} \Psi\right) \times \nabla \Psi}{\mu_{0} \rho_{0}} .
$$

Equations (4) and (7) are the basic equations to be explored in this work.

Let us assume that the plasma is ideal throughout the whole space, except in a thin slab, whose electric 
resistivity $\eta$ is a very small, although finite, number. The plasma slab extends infinitely parallel to the $y z$ plane, and from $x=-a / 2$ to $x=+a / 2$, with $a>0$. We also assume that the plasma is in a static state of equilibrium, characterized by $\Psi=\Psi(x)$ and $\Phi=$ 0 . Then, the first of (1) shows that the equilibrium magnetic field $\vec{B}=\hat{z} B_{\| 0}+\hat{y} B_{\perp}$, where $B_{\perp}=-\Psi^{\prime}$, with the prime standing for the derivative with respect to $x$. Since the current density is expected to become vanishingly small far away from the plasma slab, the second of (3) shows that $\left|\Psi^{\prime}\right|=B_{\perp 0}$, a constant, for $|x| \gg a$. Thus, we define the Alfvén speed $V_{\mathrm{A}}=$ $B_{\perp 0}\left(\mu_{0} \rho_{0}\right)^{-1 / 2}$ and time $\tau_{\mathrm{A}}=a V_{\mathrm{A}}^{-1}$ scales. Hence, we introduce the normalization $t \rightarrow \tau_{\mathrm{A}} t, \nabla \rightarrow a^{-1} \nabla$, $\Psi \rightarrow a B_{\perp 0} \Psi, \Phi \rightarrow a V_{\mathrm{A}} \Phi$, and $\chi \rightarrow a V_{\mathrm{A}} B_{\perp 0} \chi$. Given the above considerations, we obtain the dimensionless version of (4) and (7),

$$
\begin{gathered}
\hat{z} \cdot \nabla \chi+\left(\frac{\partial}{\partial t}+\vec{V} \cdot \nabla\right) \Psi=\frac{\tau_{\mathrm{A}}}{\tau_{\mathrm{D}}}\left(1+\frac{\tau_{\mathrm{C}}}{\tau_{\mathrm{A}}} \frac{\partial}{\partial t}\right) \nabla^{2} \Psi, \\
\left(\frac{\partial}{\partial t}+\vec{V} \cdot \nabla\right) \nabla^{2} \Phi=\hat{z} \cdot \nabla\left(\nabla^{2} \Psi\right) \times \nabla \Psi,
\end{gathered}
$$

respectively, where we have included the resistive diffusion time scale $\tau_{\mathrm{D}}=a^{2} \mu_{0} \eta^{-1}$. Equations (8) show that the equilibrium condition for the static state can be read as $\hat{z} \cdot \nabla \chi=\tau_{\mathrm{A}} \tau_{\mathrm{D}}^{-1} \Psi^{\prime \prime}$.

About the static state of equilibrium, we assume that the magnetic and flow fluxes are slightly perturbed in the form $\Psi(x, y ; t)=\Psi(x)+\psi(x, y) \mathrm{e}^{\gamma t}$ and $\Phi(x, y ; t)=$ $\phi(x, y) \mathrm{e}^{\gamma t}$, respectively, where $\gamma$ is a (dimensionless) time rate. By "slightly perturbed", we mean that $|\phi| \sim$ $|\psi| \ll|\Psi|$ (recall that the equilibrium $\Phi=0$ ). Then, by retaining only terms proportional to $\psi$ and $\phi$, and to their derivatives, (8) lead to the linear perturbed equations (of course, any possible perturbation on $\chi$ is also assumed to depend only on $x, y$, and $t$ )

$$
\begin{gathered}
\gamma \psi+\Psi^{\prime} \frac{\partial \phi}{\partial y}=\frac{\tau_{\mathrm{A}}}{\tau_{\mathrm{D}}}\left(1+\gamma \frac{\tau_{\mathrm{C}}}{\tau_{\mathrm{A}}}\right) \nabla^{2} \psi, \\
\gamma \nabla^{2} \phi=\left(\Psi^{\prime \prime \prime}-\Psi^{\prime} \nabla^{2}\right) \frac{\partial \psi}{\partial y},
\end{gathered}
$$

respectively.

Since the equilibrium magnetic flux depends only on the $x$-coordinate, the perturbative functions can be Fourier decomposed in the $y$-coordinate. A careful inspection of $(9)$ reveals that $\psi$ and $\phi$ exhibit opposite parities with respect to $y$. Thus, we choose to Fourier decompose the perturbative functions in the form $\psi(x, y)=\psi(x) \cos (k y)$ and $\phi(x, y)=\phi(x) \sin (k y)$, where $k$ is a (dimensionless) wavenumber. With this choice, 9 become

$$
\begin{array}{r}
\gamma \psi+k \Psi^{\prime} \phi=\frac{\tau_{\mathrm{A}}}{\tau_{\mathrm{D}}}\left(1+\gamma \frac{\tau_{\mathrm{C}}}{\tau_{\mathrm{A}}}\right)\left(\psi^{\prime \prime}-k^{2} \psi\right), \\
\gamma\left(\phi^{\prime \prime}-k^{2} \phi\right)=-k\left(\Psi^{\prime \prime \prime} \psi-\Psi^{\prime}\left(\psi^{\prime \prime}-k^{2} \psi\right)\right),
\end{array}
$$

respectively.

\subsection{Solutions in the IDEAL AND RESistive REGIONS}

In the ideal region, $\eta \rightarrow 0$ and, according to the first of $10, \phi$ is given by

$$
\phi=\frac{\gamma}{k \Psi^{\prime}}\left(\left(\frac{\delta_{\mathrm{e}}}{a}\right)^{2}\left(\psi^{\prime \prime}-k^{2} \psi\right)-\psi\right),
$$

where $\delta_{\mathrm{e}}^{2}=m_{\mathrm{e}}\left(n_{\mathrm{e}} e^{2} \mu_{0}\right)^{-1}$, with $\delta_{\mathrm{e}}$ standing for the electron skin depth. Since $\gamma$ is expected to become vanishingly small in the limit $\eta \rightarrow 0$ (this is true for tearing modes but not for kink modes, which can become unstable even in the ideal limit [33), by substituting (11) in the second of (10), we see that $\psi$ satisfies the differential equation

$$
\psi^{\prime \prime}=\left(k^{2}+\frac{\Psi^{\prime \prime \prime}}{\Psi^{\prime}}\right) \psi
$$

in the limit $\gamma \rightarrow 0$.

Except for much simplified equilibrium models, 12 must be treated numerically. However, the asymptotic behavior of its solution in the limit $x \rightarrow 0$ can be easily found by making use of the Frobenius method. To see this, we first expand the equilibrium magnetic field in a Taylor series about the origin, by noting that $\Psi^{\prime}=0$ at $x=0$. Therefore, 12 can be approximated to

$$
\psi^{\prime \prime}=\frac{\kappa}{x} \psi,
$$

where the number $\kappa$ satisfies the relation

$$
\kappa=\frac{\Psi^{\prime \prime \prime}(0)}{\Psi^{\prime \prime}(0)} .
$$

The general solution of (13) reads as [41]

$$
\psi(x)=\hat{\psi} \hat{u}(\kappa x)+\bar{\psi} \bar{u}(\kappa x),
$$

where $\hat{\psi}$ and $\bar{\psi}$ are constants, and

$$
\begin{array}{r}
\hat{u}(\kappa x)=\kappa x+\frac{1}{2}(\kappa x)^{2}+\frac{1}{12}(\kappa x)^{3}+\ldots, \\
\bar{u}(\kappa x)=1-\kappa x-\frac{5}{4}(\kappa x)^{2}-\ldots+\hat{u} \ln |\kappa x|
\end{array}
$$

provide the regular and irregular parts, respectively, of $\psi$ in the limit $x \rightarrow 0$. As it appears, about the origin, the dominant behavior of $\psi$ is given by

$$
\psi(x)=\bar{\psi}(1+\kappa x \ln |\kappa x|) .
$$

Equation (17) shows that $\psi$ is actually a constant, while its first and higher order derivatives diverge in the limit $x \rightarrow 0$. Such asymptotic behavior is the source of the so-called constant- $\psi$ approximation in the analytical theory of tearing modes.

Given the functional form (17) of the asymptotic solution of 12 about the origin, 13) shows that the logarithmic derivative of $\psi$ exhibits a jump,

$$
\Delta^{\prime}=\frac{1}{\bar{\psi}} \int_{0_{-}}^{0_{+}} \psi^{\prime \prime} d x
$$


across the resistive layer. The asymptotic solution (17) of $(12)$ in the ideal region suggests that the perturbative function $\psi$ is, to the lowest order, also a constant inside the resistive layer. However, the varying parts of $\psi$ and $\phi$ inside the resistive layer are not yet known. Actually, the characteristic length scale of the variation of the fields is not the same for both ideal and resistive phenomena. When resistivity is neglected, the magnetic field is frozen inside the plasma, and the perturbed fluid motions can cause substantial distortions in the field lines. Therefore, significant gradients of perturbed fields can ensue in the resistive layer. This is the motivation for making use of the boundary layer technique in the analytical theory of tearing modes 42 .

In order to apply the boundary layer technique to solve 10 inside the resistive layer, first we need to identify a small scaling parameter among the relevant physical quantities. For fully ionized plasmas, it is well-known that $\eta \sim T_{\mathrm{e}}^{-3 / 2}$, with $T_{\mathrm{e}}$ standing for the electron temperature [7]. For typically high values of the electron temperature, the upper-limit of the ratio of the Alfvén to the diffusion time scales is quite a small number, $\tau_{\mathrm{A}} \tau_{\mathrm{D}}^{-1}<10^{-5}[6]$. Hence, we introduce the scaling

$$
\begin{aligned}
\gamma & =\left(\frac{\tau_{\mathrm{A}}}{\tau_{\mathrm{D}}}\right)^{q} \omega, & \frac{\tau_{\mathrm{C}}}{\tau_{\mathrm{A}}} & =\left(\frac{\tau_{\mathrm{A}}}{\tau_{\mathrm{D}}}\right)^{-q} \theta, \\
x & =\left(\frac{\tau_{\mathrm{A}}}{\tau_{\mathrm{D}}}\right)^{r} \xi, & \Psi^{\prime} & =-\left(\frac{\tau_{\mathrm{A}}}{\tau_{\mathrm{D}}}\right)^{r} \xi, \\
\psi(x) & =\bar{\psi}+\left(\frac{\tau_{\mathrm{A}}}{\tau_{\mathrm{D}}}\right)^{r} \tilde{\psi}(\xi), & \phi(x) & =\left(\frac{\tau_{\mathrm{A}}}{\tau_{\mathrm{D}}}\right)^{s} \frac{\gamma}{k} \varphi(\xi),
\end{aligned}
$$

where we see that $\omega \theta=\gamma \tau_{\mathrm{C}} \tau_{A}^{-1}$, since inertial effects should be actually important at very short length scales, $\Psi^{\prime}$ vanishes linearly with $\xi$ inside the resistive layer, $\bar{\psi}$ and $\tilde{\psi}$ provide the constant and varying parts, respectively, of $\psi$ inside the resistive layer, and $\gamma k^{-1}$ is included in the definition of $\varphi$ to simplify further calculations.

Given the considerations above, we obtain the scaled version of 10 ,

$$
\begin{gathered}
\bar{\psi}+\left(\frac{\tau_{\mathrm{A}}}{\tau_{\mathrm{D}}}\right)^{r} \tilde{\psi}-\left(\frac{\tau_{\mathrm{A}}}{\tau_{\mathrm{D}}}\right)^{r+s} \xi \varphi \\
=\frac{1+\omega \theta}{\omega}\left(\frac{\tau_{\mathrm{A}}}{\tau_{\mathrm{D}}}\right)^{1-q-r}\left(\frac{d^{2} \tilde{\psi}}{d \xi^{2}}-\left(\frac{\tau_{\mathrm{A}}}{\tau_{\mathrm{D}}}\right)^{r} k^{2}\left(\bar{\psi}+\left(\frac{\tau_{\mathrm{A}}}{\tau_{\mathrm{D}}}\right)^{r} \tilde{\psi}\right)\right) \\
\frac{d^{2} \varphi}{d \xi^{2}}-\left(\frac{\tau_{\mathrm{A}}}{\tau_{\mathrm{D}}}\right)^{2 r} k^{2} \varphi \\
=-\left(\frac{\tau_{\mathrm{A}}}{\tau_{\mathrm{D}}}\right)^{-2 q+2 r-s}\left(\frac{k}{\omega}\right)^{2} \xi\left(\frac{d^{2} \tilde{\psi}}{d \xi^{2}}\right. \\
\left.-\left(\frac{\tau_{\mathrm{A}}}{\tau_{\mathrm{D}}}\right)^{r} k^{2}\left(\bar{\psi}+\left(\frac{\tau_{\mathrm{A}}}{\tau_{\mathrm{D}}}\right)^{r} \tilde{\psi}\right)\right),
\end{gathered}
$$

respectively.

The powers $q, r$, and $s$ must be chosen by requiring that the terms proportional to $\xi$ and to the second derivative of $\tilde{\psi}$ become of the same order of $\bar{\psi}$ (the constant- $\psi$ approximation). This is achieved by choosing $r+s=0,1-q-r=0$, and $-2 q+2 r-s=0$. Then, $q=3 / 5, r=2 / 5$, and $s=-2 / 5$. Thus, 20 become

$$
\begin{aligned}
& \bar{\psi}+\left(\frac{\tau_{\mathrm{A}}}{\tau_{\mathrm{D}}}\right)^{2 / 5} \tilde{\psi}-\xi \varphi \\
& =\frac{1+\omega \theta}{\omega}\left(\frac{d^{2} \tilde{\psi}}{d \xi^{2}}-\left(\frac{\tau_{\mathrm{A}}}{\tau_{\mathrm{D}}}\right)^{2 / 5} k^{2}\left(\bar{\psi}+\left(\frac{\tau_{\mathrm{A}}}{\tau_{\mathrm{D}}}\right)^{2 / 5} \tilde{\psi}\right)\right) \\
& \frac{d^{2} \varphi}{d \xi^{2}}-\left(\frac{\tau_{\mathrm{A}}}{\tau_{\mathrm{D}}}\right)^{4 / 5} k^{2} \varphi \\
& =-\left(\frac{k}{\omega}\right)^{2} \xi\left(\frac{d^{2} \tilde{\psi}}{d \xi^{2}}-\left(\frac{\tau_{\mathrm{A}}}{\tau_{\mathrm{D}}}\right)^{2 / 5} k^{2}\left(\bar{\psi}+\left(\frac{\tau_{\mathrm{A}}}{\tau_{\mathrm{D}}}\right)^{2 / 5} \tilde{\psi}\right)\right)
\end{aligned}
$$

respectively.

By retaining only terms proportional to the small scaling parameter $\tau_{\mathrm{A}} \tau_{\mathrm{D}}^{-1}, 21$ approach

$$
\begin{gathered}
\bar{\psi}-\xi \varphi=\frac{1+\omega \theta}{\omega} \frac{d^{2} \tilde{\psi}}{d \xi^{2}}, \\
\frac{d^{2} \varphi}{d \xi^{2}}=-\left(\frac{k}{\omega}\right)^{2} \xi \frac{d^{2} \tilde{\psi}}{d \xi^{2}}
\end{gathered}
$$

respectively. By combining 22, we obtain

$$
\frac{d^{2} \varphi}{d \xi^{2}}=-\frac{k^{2}}{(1+\omega \theta) \omega} \xi(\bar{\psi}-\xi \varphi) .
$$

In order to solve (23), it proves useful to introduce the transformations

$$
\begin{gathered}
\xi=\left(\frac{(1+\omega \theta) \omega}{k^{2}}\right)^{1 / 4} \zeta, \\
\varphi(\xi)=-\left(\frac{k^{2}}{(1+\omega \theta) \omega}\right)^{1 / 4} \bar{\psi} f(\zeta) .
\end{gathered}
$$

By substituting (24) in 23), we obtain

$$
\frac{d^{2} f}{d \zeta^{2}}=\zeta(1+\zeta f)
$$

The solution of 25 can be written in terms of the integral representation [43, 44]

$$
f(\zeta)=-\zeta \int_{0}^{1 / 2} \frac{1}{\left(1-4 \beta^{2}\right)^{1 / 4}} \mathrm{e}^{-\zeta^{2} \beta} d \beta
$$

which implies the asymptotic behavior

$$
f(\zeta)=-\frac{1}{\zeta}-\frac{2}{\zeta^{5}}-\ldots
$$

in the limit $|\zeta| \gg 1$.

\subsection{Asymptotic matching AND GRowth RATE}

In order to asymptotically match the solutions of 10 in the ideal region and inside the resistive layer, it is 
sufficient to identify 18 with the jump in the logarithmic derivative of the scaled $\psi$ across the resistive layer, to the lowest order. Thus, given the scaling $(19)$, we identify

$$
\Delta^{\prime}=\frac{1}{\bar{\psi}} \int_{-w / 2}^{+w / 2} \frac{d^{2} \tilde{\psi}}{d \xi^{2}} d \xi
$$

where $w>0$ is the (scaled, dimensionless) width of the resistive layer. By combining the second of 22 with (28), we obtain

$$
\Delta^{\prime}=-\left(\frac{\omega}{k}\right)^{2} \frac{1}{\bar{\psi}} \int_{-w / 2}^{+w / 2} \frac{d^{2} \varphi}{d \xi^{2}} \frac{d \xi}{\xi} .
$$

By substituting (24) in 29, we obtain

$$
\Delta^{\prime}=\frac{\omega^{5 / 4}}{k^{1 / 2}(1+\omega \theta)^{3 / 4}} \int_{-\infty}^{+\infty} \frac{d^{2} f}{d \zeta^{2}} \frac{d \zeta}{\zeta},
$$

where we have extended the limits of the integral to $\pm \infty$ because the integrand $\sim-\zeta^{-4}$ inside the resistive layer, according to the asymptotic behavior of $f$ in the limit $|\zeta| \gg 1$, as given by (27). Actually, in accordance with 26 , the integral in 30 can be written as

$$
\begin{aligned}
& \int_{\zeta=-\infty}^{\zeta=+\infty} \frac{d^{2} f}{d \zeta^{2}} \frac{d \zeta}{\zeta} \\
= & 2 \int_{\zeta=-\infty}^{\zeta=+\infty} d \zeta \int_{\beta=0}^{\beta=1 / 2} \frac{3 \beta-2 \zeta^{2} \beta^{2}}{\left(1-4 \beta^{2}\right)^{1 / 4}} \mathrm{e}^{-\zeta^{2} \beta} d \beta
\end{aligned}
$$

Since the integral (31) can be expressed in terms of Gamma functions [41, 30 finally yields the sought identification,

$$
\Delta^{\prime}=\frac{2 \pi \Gamma(3 / 4)}{k^{1 / 2} \Gamma(1 / 4)} \frac{\omega^{5 / 4}}{(1+\omega \theta)^{3 / 4}} .
$$

On the assumption that $\Delta^{\prime}$ is known, (32) implies

$$
\frac{\omega^{5 / 4}}{(1+\omega \theta)^{3 / 4}}=\frac{\Delta^{\prime} \Gamma(1 / 4)}{2 \pi \Gamma(3 / 4)} k^{1 / 2} .
$$

Therefore, according to the scaling 19 , 33 can be written as

$$
\frac{\gamma^{5 / 4}}{\left(1+\gamma \tau_{\mathrm{C}} \tau_{\mathrm{A}}^{-1}\right)^{3 / 4}}=\frac{\Delta^{\prime} \Gamma(1 / 4)}{2 \pi \Gamma(3 / 4)} k^{1 / 2} \tau_{\mathrm{A}}^{3 / 4} \tau_{\mathrm{D}}^{-3 / 4},
$$

in terms of dimensionless quantities. By plugging back the actual physical quantities in (34), we finally read

$$
\frac{\gamma^{5 / 4}}{\left(1+\gamma \tau_{\mathrm{C}}\right)^{3 / 4}}=\frac{\Delta^{\prime} a \Gamma(1 / 4)}{2 \pi \Gamma(3 / 4)}(k a)^{1 / 2} \tau_{\mathrm{A}}^{-1 / 2} \tau_{\mathrm{D}}^{-3 / 4} \text {. }
$$

Equation 35 shows that if $\Delta^{\prime}>0$, then $\gamma>0$, and the aforementioned static state of equilibrium becomes unstable to the linear perturbation. In particular, for sufficiently long perturbative wavelengths, inertial effects due to charged species are negligible, $\gamma \tau_{\mathrm{C}} \ll 1$, and (35) simplifies to

$$
\gamma=\left(\frac{\Delta^{\prime} a \Gamma(1 / 4)}{2 \pi \Gamma(3 / 4)}\right)^{4 / 5}(k a)^{2 / 5} \tau_{\mathrm{A}}^{-2 / 5} \tau_{\mathrm{D}}^{-3 / 5} .
$$

Equation 36 shows the standard result of the analytical theory of tearing modes, which establishes that the growth rate $\gamma$ scales with the plasma resistivity $\eta$ as $\gamma \sim \eta^{3 / 5}$ [30.

\section{Growth Rate amplification}

Now we get to the main result of this work. For sufficiently short wavelengths, inertial effects can become important. Then, in the limit $\gamma \tau_{\mathrm{C}} \gg 1$, from (35), we find that

$$
\gamma=\left(\frac{\Delta^{\prime} a \Gamma(1 / 4)}{2 \pi \Gamma(3 / 4)}\right)^{2}(k a) \tau_{\mathrm{A}}^{-1}\left(\frac{\delta_{\mathrm{e}}}{a}\right)^{3} .
$$

Equation (37) shows that inertial effects can provoke quite a significant change on the scaling of the growth rate with the relevant plasma parameters. As a matter of fact, we see that $\gamma$ does not depend on the plasma resistivity any longer. Actually, it scales now with the electron number density as $\gamma \sim n_{\mathrm{e}}^{-3 / 2}$.

Beyond the above mentioned qualitative result, can we quantify the change of the growth rate due to a change on the perturbative wavelength? To answer this question, first we observe that the product $\Delta^{\prime} a$, in the general equation (35), is a function of the product $k a$ [45,49]. Perhaps, the most illustrative example of this issue is provided by the so-called Harris model, which assumes the profile 50

$$
\vec{J}=\hat{z} \frac{B_{\perp 0}}{a \mu_{0}} \operatorname{sech}^{2} \frac{x}{a}
$$

for the equilibrium current density. By substituting (38) in the second of (3), one can calculate the equilibrium magnetic flux. Next, by substituting the latter in (12), one can compute the perturbative magnetic flux. Finally, (18) yields the well-known result

$$
\Delta^{\prime} a=2\left(\frac{1}{k a}-k a\right)
$$

Given the above considerations, let us assume that two plane resistive slabs are formed in an infinite ideal plasma. The two slabs have the same electric resistivity and are subjected to the same equilibrium magnetic field. The only difference between them is their thicknesses. Thus, we can explore the situation for which the product $k a$ is the same for both slabs. This means that the thicker slab can accommodate a longer perturbative wavelength, which we call $\Lambda$, and the thinner slab can accommodate a shorter perturbative wavelength, which we call $\lambda$. Therefore, from 35 , we find that

$$
\frac{\left(\Gamma \tau_{\mathrm{C}}\right)^{1 / 2}}{\left(\gamma \tau_{\mathrm{C}}\right)^{5 / 4}}=\left(\frac{\lambda}{\Lambda}\right)^{-2}
$$

where the growth rates $\gamma$ and $\Gamma$ are assumed to satisfy the conditions $\gamma \tau_{\mathrm{C}} \ll 1$ and $\Gamma \tau_{\mathrm{C}} \gg 1$, respectively. To see the consequences of result 40 , suppose that $\gamma \tau_{\mathrm{C}} \sim 10^{-2}$ for the unstable mode with the longer perturbative wavelength $\Lambda$. Hence, if the latter is moderately shortened, for instance, to $\lambda \sim 10^{-2} \Lambda$, then 
(40) shows that $\Gamma \tau_{\mathrm{C}} \sim 10^{3}$. This means that the standard growth rate $\gamma$ of the tearing mode is amplificated to $\Gamma \sim 10^{5} \gamma$, quite a significant amplification due to inertial effects of charged species in the plasma.

\section{Conclusion}

In this work, we have explored the influence of perturbative wavelengths, shorter than those usually considered, on the growth rate $\gamma$ of the tearing modes. Thus, we have adopted an extended form of Ohm's law, which includes a finite relaxation time for the current density, due to inertial effects of charged species. In the long wavelength limit, we have observed the standard $\gamma$ of the tearing modes. However, in the short wavelength limit, we have shown that $\gamma$ does not depend on the fluid resistivity any longer. Actually, we have found out that $\gamma$ now scales with the electron number density $n_{\mathrm{e}}$ as $\gamma \sim n_{\mathrm{e}}^{-3 / 2}$. Therefore, through a suitable combination of both limiting results, we have shown that the standard $\gamma$ can be substantially amplificated, even by moderate shortenings of perturbative wavelengths. Further developments of our theory may contribute to the explanation of the fast magnetic reconnection of field lines, as observed in astrophysical plasmas.

\section{REFERENCES}

[1] I. B. Bernstein, E. A. Frieman, M. D. Kruskal, R. M. Kulsrud. Proc R Soc A244:17, 1958.

[2] W. A. Newcomb. Ann Phys 10:232, 1960.

[3] J. M. Greene, J. L. Johnson. Phys Fluids 5:510, 1962.

[4] R. J. Goldston, P. H. Rutherford. Introduction to Plasma Physics. Institute of Physics, Bristol, 2000.

[5] K. Nishikawa, M. Wakatani. Plasma Physics: Basic Theory with Fusion Applications. Third edition. Springer-Verlag, Berlin, 2000.

[6] K. Miyamoto. Plasma Physics and Controlled Nuclear Fusion. Springer-Verlag, Berlin, 2005.

[7] L. Spitzer. Physics of Fully Ionized Gases. Second edition. Dover Publications, New York, 2006.

[8] J. P. Freidberg. Ideal MHD. Cambridge University Press, Cambridge, 2014.

[9] V. D. Shafranov. Sov Phys Tech Phys 15:175, 1970.

[10] D. F. Düchs, H. P. Furth, P. H. Rutherford. Nucl Fusion 12:341, 1972.

[11] J. G. Cordey, F. A. Haas. Nucl Fusion 16:605, 1976.

[12] C. C. Grimes, G. Adams. Phys Rev Lett 36:145, 1976.

[13] M. J. Forrest, P. G. Carolan, N. J. Peacock. Nature 271:718, 1978.

[14] P. H. Rutherford. Phys Fluids 16:1903, 1973.

[15] T. H. Stix. Phys Rev Lett 36:521, 1976.

[16] B. V. Waddell, B. Carreras, H. R. Hicks, J. A. Holmes. Phys Fluids 22:896, 1979.

[17] B. A. Carreras, P. W. Gaffney, H. R. Hicks, J. D. Callan. Phys Fluids 25:1231, 1982.

[18] S. J. Zweben, R. W. Gould. Nucl Fusion 25:171, 1985.
[19] R. W. White, D. A. Monticello, M. N. Rosenbluth, B. V. Waddell. Phys Fluids 20:800, 1977.

[20] M. F. Turner, J. Wesson. Nucl Fusion 22:1069, 1982.

[21] A. I. Smolyakov. Sov J Plasma Phys 15:667, 1989.

[22] A. J. Wootton, B. A. Carreras, H. Matsumoto, K. McGuire, W. A. Peebles, Ch. P. Ritz, P. W. Terry, S. J. Zweben. Phys Fluids B2:2879, 1990.

[23] B. Kadomtsev. Nucl Fusion 31:1301, 1991.

[24] E. N. Parker. J Geophys Res 62:509, 1957.

[25] D. Biskamp. Phys Fluids 29:1520, 1986.

[26] P. Goldreich, S. Sridhar. Astrophys J 438:763, 1995.

[27] A. Lazarian, E. Vishniac. Astrophys J 517:700, 1999.

[28] G. Kowal, A. Lazarian, E. Vishniac, K. Otmianowska-Mazur. Astrophys J 700:63, 2009.

[29] G. Kowal, A. Lazarian, E. Vishniac, K. OtmianowskaMazur. Nonlinear Proc Geoph 19:297, 2012.

[30] H. P. Furth, J. Killeen, M. N. Rosenbluth. Phys Fluids 6:459, 1963.

[31] B. Coppi, J. M. Greene, J. L. Johnson. Nucl Fusion 6:101, 1966.

[32] A. H. Glasser, J. M. Greene, J. L. Johnson. Phys Fluids 18:875, 1975.

[33] B. Coppi, R. M. O. Galvão, R. Pellat, M. N. Rosenbluth, P. H. Rutherford. Sov J Plasma Phys 2: 533, 1976.

[34] A. M. M. Todd, M. S. Chance, J. M. Greene, R. C. Grimm, J. L. Johnson, J. Manickam. Phys Rev Lett 38:826, 1977.

[35] J. P. Mondt, J. Weiland. J Plasma Phys 34:143, 1985.

[36] F. E. M. Silveira. J Phys: Conf Ser 370:012005, 2012.

[37] F. E. M. Silveira. J Plasma Phys 79:45, 2013.

[38] F. E. M. Silveira, R. M. O. Galvão. Phys Plasmas 20:082126, 2013.

[39] F. E. M. Silveira, H. I. Orlandi. Phys Plasmas 23:042111, 2016.

[40] F. E. M. Silveira. Plasma Phys Tech 3:155, 2016.

[41] H. Jeffreys, B. S. Jeffreys. Methods of Mathematical Physics. Third edition. Cambridge University Press, Cambridge, 1999.

[42] C. M. Bender, S. A. Orszag. Advanced Mathematical Methods for Scientists and Engineers. Vol. 1: Asymptotic Methods and Perturbation Theory. Springer-Verlag, New York, 1999.

[43] G. L. Johnston. J Math Phys 19:635, 1978.

[44] R. M. O. Galvão. Physica 122C:289, 1983.

[45] B. Bertotti. Ann Phys 25:271, 1963.

[46] M. G. Haines. Nucl Fusion 17:811, 1977.

[47] J. N. Leboeuf, T. Tajima, J. M. Dawson. Phys Fluids 25:784, 1982.

[48] Y. Ono, M. Yamada, T. Akao, T. Tajima, R. Matsumoto. Phys Rev Lett 76:3328, 1996.

[49] B. V. Somov, T. Kosugi. Astrophys J 485:859, 1997.

[50] E. G. Harris. Nuovo Cimento 23:115, 1962. 